\title{
La recuperación de los colores originales de las Torres de Quart de Valencia
}

\author{
Ana Torres Barchino ${ }^{\mathrm{a}}$, Juan Serra lluch ${ }^{\mathrm{b}}$, Jorge Llopis Verdúc José Luis Higón Calvet $^{\mathrm{d}}$, \\ Begoña Saiz Mauleón ${ }^{\mathrm{e}}$, Ramón Villaplana Guillén ${ }^{\mathrm{f}}$ \\ ${ }^{a}$ Universidad Politécnica de Valencia, España, atorresb@ega.upv.es, bjuanserra@ega.upv.es, cjllopis@ega.upv.es, \\ jihigonc@ega.upv.es, ${ }^{\mathrm{e} b s a i z m a @ e g a . u p v . e s,}{ }^{\mathrm{f}}$ rvillapl@ega.upv.es
}

\begin{abstract}
"The communication presents the methodology and conclusions of the study developed by the Color Group of the Instituto Universitario de Restauración del Patrimonio of the Universitat Politècnica de València, in the context of the restoration of the Torres de Quart of Valencia (XV century). In this intervention, it was conducted a comprehensive study of the original coatings, both regarding to their mineral composition and their colors. This study included the determination of the original color chemical characteristics, an exhaustive physical color measurement of the remnants of pigments on the outer facades, over three coating layers with different nature, and also a study of the color degradation conditions in the Torres de Quart and their relation to the sunlight exposure ${ }^{1}$.
\end{abstract}

Keywords: Patrimonio, Restauración, Color, Mortero.

\section{Introducción}

El proyecto de restauración de las Torres de Quart es una actuación promovida por la Concejalía de Cultura del Excmo. Ayuntamiento de Valencia desarrollado durante el año 2007. El proyecto fue coordinado por los arquitectos D. Manuel Ramírez y D. Javier Benlloch, participando el Grupo de Color, Fotogrametría, Levantamientos y Análisis Gráfico para la Arquitectura histórica, del Instituto de restauración del Patrimonio de la Universidad Politécnica de Valencia, en la determinación de las características cromáticas originales, así como un estudio de las condiciones de degradación cromática al que estaban expuestas las Torres de Quart y su relación con el soleamiento, analizando la degradación del mortero original en relación con la diferente exposición solar de cada cara, con el objetivo de desarrollar una propuesta de actuación que respetase el cromatismo original, y dotase a la intervención de unas condiciones de durabilidad suficientes.

Las Torres de Quart constituyen uno de los últimos vestigios del recinto amurallado de Valencia, construido en el año 1400. El diseño original de la obra, característico de la avanzada experimentación geométrica característica de la estereotomía de las fábricas petreas del panorama arquitectónico valenciano de mediados del s. XV, es obra de Francesc Baldomar, pero su construcción se dilata en el tiempo, siendo Pere Bonfill quien finaliza su construcción en 1469 (Zaragozá, 2007). El edificio se compone de tres cuerpos arquitectónicos claramente diferenciados: la torre norte, el portal de acceso y la torre sur. Característica del edificio es el esviaje de las torres, que no se resuelven ortogonalmente a la muralla, sino que se alinean con el eje del antiguo camino que conduce a la población de 
Quart de Poblet, topónimo que da nombre a este portal de acceso a la ciudad.

El sistema constructivo consta de gruesos muros de tapial enlucidos con mortero liso de cal y sillares de piedra en los ángulos, aristas, bóvedas y coronación. Sus almenas se reconstruyen en piedra caliza de Godella en la rehabilitación llevada a cabo por D. Carlos Soler en los años cincuenta. En la última restauración de por Emilio Rieta y Román Jiménez (1976-1982), se construye la monumental escalinata exterior con sillares procedentes del palacio de los condes de Parcent. Ninguno de los volúmenes que componen el conjunto presenta restos de color en las fachadas orientadas hacia la ciudad. En dichas fachadas intramuros existen grandes vanos que permiten visualizar el color de los espacios interiores, pero su exterior carece de pigmentación. Las fachadas extramuros, sin embargo, están revestidas con dos tipos de mortero que en su momento se encontraban coloreados, y es en ellas donde se realizan las labores de identificación cromática.

\section{Análisis material y cromático de la superficie muraria}

En el estado previo al inicio de los trabajos, la superficie muraría del monumento se caracterizaba por las heterogéneas condiciones de conservación de la misma en función de la altura, orientación y otras condiciones concretas de exposición a los diferentes agentes susceptibles de provocar la pérdida de las características cromáticas originales. $\mathrm{Si}$ a esto unimos la enorme superficie muraría objeto de análisis, cabe concluir que la metodología de evaluación y medición para la determinación del color original, no pueda ser llevada a cabo a partir de una metodología clásica de medición puntual en los lugares mejor conservados, siendo imprescindible el desarrollo de una metodología de medición de carácter estadístico. Es por ello que se estableció una estrategia de carácter "zonal", subdividiendo la superficie total en áreas de trabajo en las que calcular la media de las mediciones cromáticas, entendiendo dicho valor resultante como una aproximación suficiente al color global de toda el área
Para ello se determinó una subdivisión de la superficie mural extramuros en paños de 1 x 1,4 $\mathrm{m} 2$, lo que generó una estructura total de 1.664 paños de control cromático. Las dimensiones elegidas para el desarrollo de esta estrategia no son arbitrarias, ya que se adecuaban correctamente a la modulación del sistema de andamiajes erigido en obra, lo que facilita el proceso de medición en su conjunto desde el punto de vista práctico, al tiempo que generaban paños de medición de una superficie que se correspondía suficientemente con las condiciones efectivas de los paños a analizar, ya que una mayor subdivisión habría provocado la existencia de notorias heterogeneidades en el estado de conservación del revestimiento en el interior de cada paño de medición.

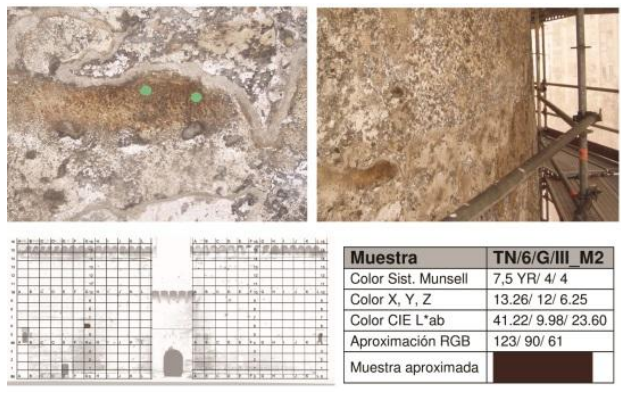

Fig. 1. Identificación de dos muestras en la Torre Norte, altura 6, cuadrante G, sección III, sobre el mortero 2 .

Para el desarrollo de la medición se partió de la hipótesis de considerar cada cuadrante establecido como una unidad cromática uniforme e indivisible a la que corresponden, por tanto, unos parámetros colorimétricos únicos. $\mathrm{La}$ asignación de los valores cromáticos de cada uno de los paneles propuestos, genera una visión global del estado cromático del conjunto de los volúmenes que componen el monumento, la tiempo que nos permite analizar si existe una relación entre la degradación cromática y parámetros tales como la orientación respecto al sol (y consecuente con la intensidad de la radiación solar soportada), su posición relativa respecto a la calle (mayor o menor exposición a las agresiones del tráfico...), y su posición relativa respecto a las diferentes tecnologías 
materiales de las torres (paramento murario de piedra...).

Respecto al proceso de de medición hay que decir que la distribución de los fragmentos cromáticos conservados no es isótropa y por tanto el número de medidas realizadas en cada cuadrante es desigual, obteniéndose un número medio de 2,8 muestras en cada uno, con una variación notable en función de la altura del cuadrante y de la orientación del mismo.

El estudio de la composición físico-química de los morteros y de las capas de policromía se ha realizado mediante Difracción de Rayos-X (DRX), Microscopía Óptica (MO) y Microscopía Electrónica de Barrido con microanálisis por dispersión de energías de rayos-X (MEB/EDX). El mortero M1 se identifica como un mortero de cal con áridos de sílice, coloreado en superficie con tierras naturales. El difractograma de rayos-X de la muestra QM6, (Fig. 4), procedente de la torre sur y extraída a nivel de mortero M1, determina como compuestos mayoritarios del mortero a la calcita y el cuarzo, con presencia minoritaria de yeso de neoformación. Estos resultados se corroboran en el microanálisis de rayos- $\mathrm{X}$ realizado en MEB/EDX de la muestra QM2 (Fig. 2).

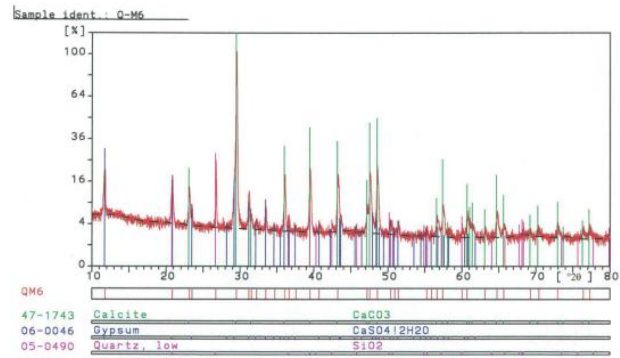

Fig. 2. Difractograma de los compuestos cristalinos mayoritarios de la muestra analizada. Muestra QM6

Los restos de pintura analizados a nivel de mortero M1, procedentes de la muestra QM2, muestran la presencia de tierras a base de alumino-silicatos y óxidos de hierro, no apreciándose coloración en masa, (Figs. 3, 4 y $5)$.

Las mediciones cromáticas se han llevado a cabo empleando un espectrofotómetro de contacto modelo Kónica Minolta CM-2600d, con sistema de iluminación esférico $\mathrm{d} / 8^{\circ}$, iluminante estándar CIE D65, reflectancia especular excluida (SCE), área de medida de $\emptyset=8 \mathrm{~mm}$ y sistema de notación del color Munsell.

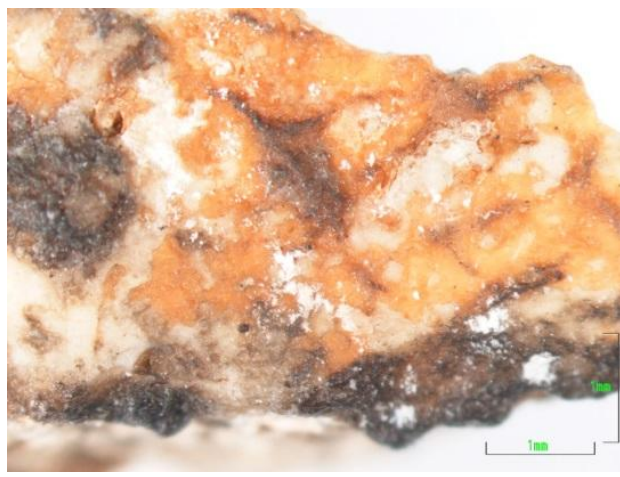

Fig. 3. Superficie de la muestra bajo lupa binocular

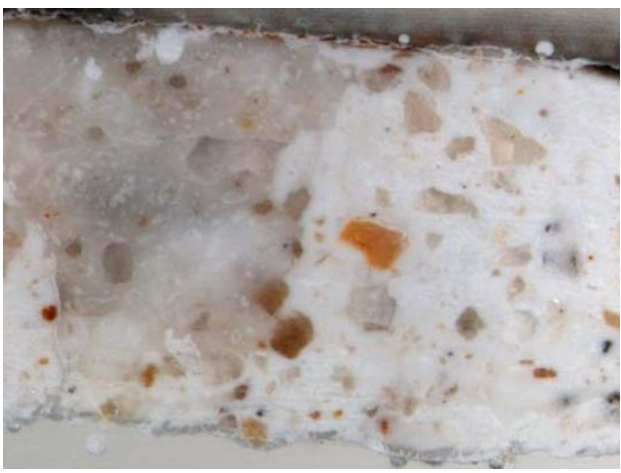

Fig. 4. Sección transversal de la muestra a 45X

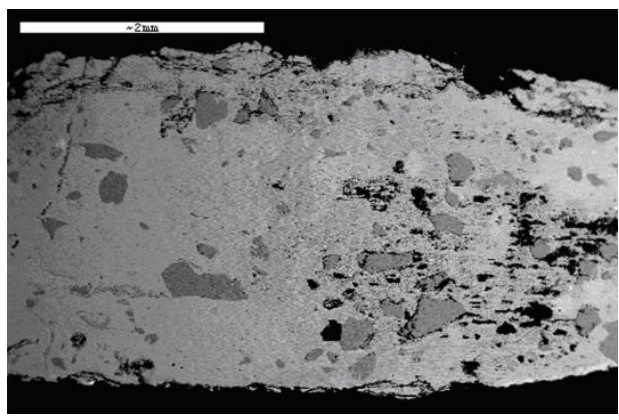

Fig. 5. Microfotografía obtenida por MEB a 25X

El tratamiento informático de la información cromática obtenida se ha desarrollado mediante el empleo del software Spectramagic NX, de la casa Kónica Minolta. 


\begin{tabular}{|c|c|c|c|c|c|}
\hline $\begin{array}{l}\text { Distribución } \\
\text { de las } \\
\text { muestras }\end{array}$ & $\frac{1,10}{\text { Torre Norte }}$ & 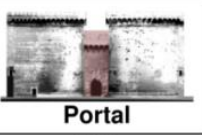 & $\frac{1.10}{\text { Torre Sur }}$ & $\frac{\text { Ins }}{\text { Pisos superiores }}$ & $\frac{\text { Pisos inferiores }}{\text { Pistes }}$ \\
\hline Mortero 1 & $74 \%$ & $0 \%$ & $26 \%$ & $24 \%$ & $76 \%$ \\
\hline Mortero 2 & $24 \%$ & $0 \%$ & $76 \%$ & $52 \%$ & $48 \%$ \\
\hline Piedra & $35 \%$ & $47 \%$ & $17 \%$ & $49 \%$ & $51 \%$ \\
\hline
\end{tabular}

Fig. 7. Proporción de color según materiales y ubicación

Respecto a las características de las capas originales de mortero, hay que decir que los restos de pigmentos encontrados se ubican sobre 3 tipos de materiales diferenciados: piedra de caliza (en adelante piedra) y dos niveles de mortero de cal de distinta antigüedad (en adelante M1 y M2). Por ello, las medidas de color se diferencian según hayan sido tomadas en cada uno de estos tres estratos.

Con posterioridad, a esta división zonal inicial se le combinará una segunda clasificación en la que esta organización "material" se combinará con los resultados del análisis de exposición a las condiciones de exposición solar.

El resultado de esta fase del estudio permite decir que la mayor concentración de pigmento en el mortero 1 (M1) se produce en la torre Norte, la mayor concentración de pigmento en el mortero 2 de (M2) corresponde a la torre sur y la piedra coloreada es más significativa en el portal. En sentido vertical la distribución es sensiblemente uniforme en $M 2$ y piedra, mientras que en $M 1$ es significativamente mayor en pisos inferiores (Fig. 6)

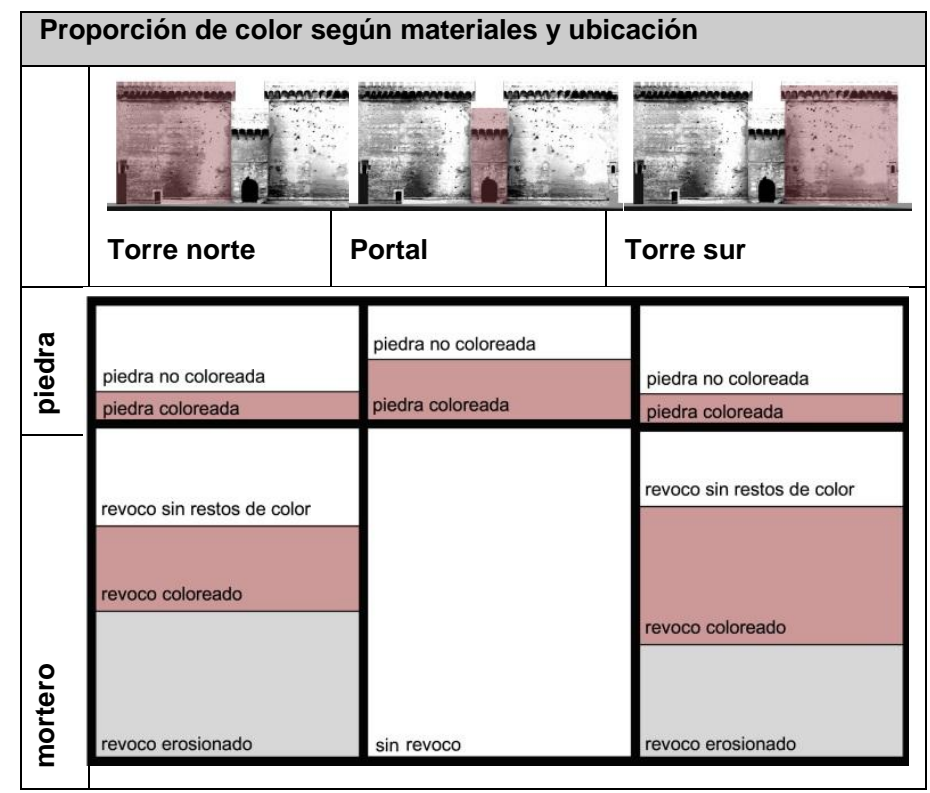

\begin{tabular}{|l|l|}
\hline & Presencia de color \\
\hline $\begin{array}{l}\text { Material } \\
\text { deteriorado }\end{array}$ \\
\hline & Ausencia de color \\
\hline
\end{tabular}

Fig. 6. Distribución de las muestras a lo largo de la fachada extramuros

De la superficie total de la fachada extramuros se observa que no existe color en ninguno de los sillares de la barbacana almenada que remata el edificio, ya que se trata de una reconstrucción de la original llevada a cabo a finales del XVIII. Lo mismo ocurre con el basamento de apoyo de ambas torres, que corresponde a una intervención posterior y carece así mismo de pigmento. Los restos arqueológicos han evidenciado que, en su origen, las torres se prolongaban bajo tierra conformando un foso actualmente colmatado. En ninguno de estos 
sillares, al igual que en las fajas de las esquinas y en la fachada intramuros, se encuentra pigmento alguno.

Los sillares que sí presentan restos de color son aquellos correspondientes al pórtico central y a las superficies protegidas bajo las bóvedas de las barbacanas de coronación.

De la superficie total de fachada enfoscada originalmente $(1.540 \mathrm{~m} 2)$, se conserva el mortero en $930,94 \mathrm{~m} 2$; lo que supone un deterioro físico del $40 \%$ de su superficie, mientras que los 769 restos de pigmento encontrados sobre los morteros permiten deducir la información cromática del $47 \%$ del enfoscado conservado $(438,2 \mathrm{~m} 2)$

Las tonalidades dominantes en el estrato M1 corresponden a la familia de colores en notación Munsell 8 YR, en la que se observa una concentración del $32 \%$ de las muestras. Le siguen las familias 5,5 YR, 10YR y otras gamas propias de las familias YR, en menor proporción (Figs. 8, 9).

El nivel M2 es el estrato coloreado más superficial, y por tanto el más actual. Recubre la mayor parte de la fachada extramuros y cuenta con una superficie significativa de restos cromáticos, lo que ha permitido hacer una medición muy extensa en número de muestras. Las tonalidades dominantes en el estrato M2 corresponden a la familia de colores en notación Munsell 7,5 YR, en la que se observa una concentración del $46 \%$ de las muestras. Le siguen las familias $10 \mathrm{YR}, 8,5 \mathrm{YR}$ y otras gamas propias de las familias YR en mucha menor proporción.

Así mismo, sobre el estrato "piedra" se han encontrado restos de pinturas aplicadas "in situ", que ocultaban el color natural propio del material. Las tonalidades dominantes sobre la piedra corresponden a la familia de colores en notación Munsell 7,5 YR, en la que se observa una concentración del 38\% de las muestras. Le siguen las familias 7 YR, 6,5 YR, 6 YR y otras gamas propias de las familias $\mathrm{YR}$ en menor proporción.
Finalmente, hay que decir que las tonalidades dominantes sobre la piedra de las barbacanas corresponden a las familias de colores en notación Munsell 7,5 YR; 8 YR; 8,5 YR; 9 YR en la que se observa una concentración del $74 \%$ de las muestras. Le siguen las familias 7 YR y otras gamas propias de las familias YR en mucha menor proporción.

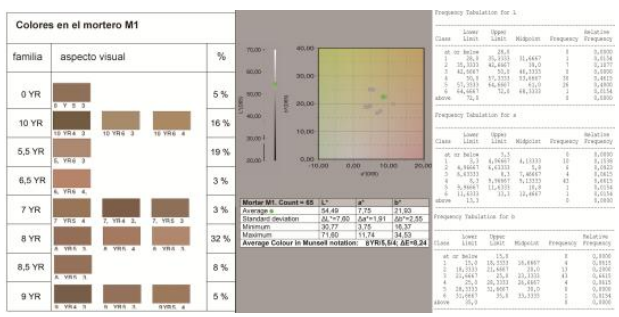

Fig. 8. Proporción de colores en mortero M1 por familias de color en notación Munsell.

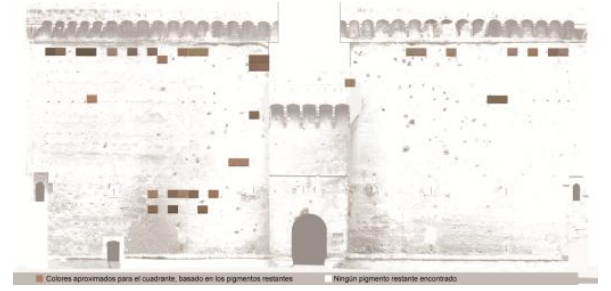

Fig. 9. Aproximación de colores en mortero M1 a lo largo de la fachada extramuros

\section{Medición del soleamiento}

De forma simultánea al proceso de identificación de los restos pigmentados se desarrolla un modelo digital para el análisis de la incidencia de la luz solar sobre las fachadas del monumento objeto de estudio. Se pretende el cálculo de la cantidad de horas de sol que recibe cada punto de las superficies extramuros de las Torres de Quart a lo largo de todo un año (Higón, Ferrer; 2003). Para ello, se recurre a la confección de cinco "mapas de sombras", lo que permite calcular el llamado Factor de Obstrucción Solar. Dicho valor escalar genera un campo sobre las superficies expuestas al sol, de tal modo que puede adquirir un valor numérico comprendido entre 0 (punto siempre iluminado) y 1 (punto siempre en sombra). El producto resultante de multiplicar el Factor de Obstrucción por el número máximo anual de horas de sol permite 
obtener, para cada punto de la superficie del modelo estudiado, la duración del periodo de incidencia solar (Fig.10).

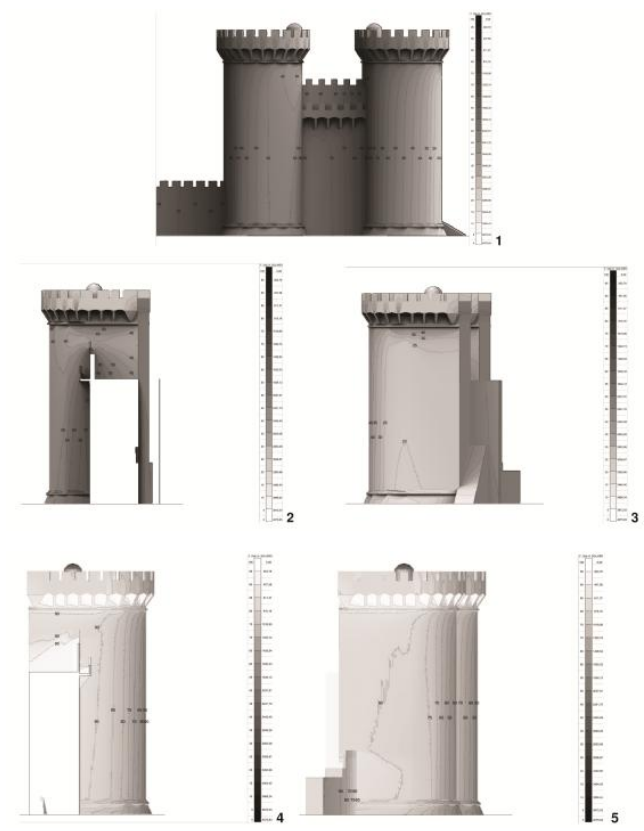

Fig. 10. Mapas de Sombras. 1-Alzado del Portal de acceso. 2.-Sección por el portal en dirección Norte. 3.-Alzado de la Torre Sur. . 4.-Sección por el Portal en dirección Sur. 5.-Alzado de la Torre Norte

\section{Análisis de los resultados}

Como resultado de este análisis de los restos de pigmentos encontrados en las fachadas extramuros de las Torres de Quart de Valencia, sobre tres estratos materiales de distinta naturaleza (mortero 1, mortero 2 y piedra), y el efecto del soleamiento sobre sus parámetros cromáticos, se ha obtenido un documento gráfico preciso del estado del color y su iluminación previo a la intervención arquitectónica.

Respecto a la caracterización de los colores en las fachadas exteriores de las Torres, esta investigación concluye que los pigmentos existentes son colores terrosos, consistentes en una mezcla de amarillo, con contenido de primario rojo en menor proporción (con predominio de las familias de color Munsell 7YR a 8 YR), con luminosidad media y poca saturación, para cada estrato material. Estos resultados son coherentes con investigaciones previas sobre el color en los revocos antiguos de los edificios del centro histórico de Valencia.

Los menores contenidos absoluto y relativo de primario rojo $\left(a^{*}\right)$ y amarillo $\left(b^{*}\right)$ se encuentran en las barbacanas, que se han visto menos expuestas a la luz solar que otros materiales, mientras que el mortero M2 y la piedra, con más incidencia solar, presentan los valores mayores de contenido absoluto y relativo de $\mathrm{a}^{*} \mathrm{y} \mathrm{b}^{*}$. Dado que estadísticamente se ha determinado la inexistencia de una relación directa entre los valores de las variables cromáticas estudiadas sobre la superficie extramuros con la diferente exposición al sol, cabe exponer la hipótesis de que la preservación del color en las barbacanas es producto no tanto de la exposición solar como de la menor exposición a otros agentes agresivos propios de la circulación, polución, agresión de viandantes, etc. Además, se han comparado los parámetros cromáticos con el factor de obstrucción solar $(\mathrm{F})$, que evalúa la exposición a la luz solar, para cada estrato material, y no se ha encontrado ninguna correlación lineal significativa entre ambos: parámetros de color y exposición solar. Las variaciones en el contenido de primarios rojo/amarillo, la claridad del color o su croma, deben haber sido causadas por otros agentes de deterioro, pero no por la cantidad de luz recibida a lo largo de los años en las fachadas exteriores de las Torres de Quart, como podía esperarse a priori. La incidencia de la luz solar no ha sido un agente de deterioro significativo en el color de las fachadas exteriores de las Torres de Quart.

El estudio demuestra que en el caso de las Torres de Quart y de los revocos coloreados que constituyen sus capas superficiales de acabado, la influencia de la exposición solar, fruto de la diferente ubicación de sus paramentos, ha sido mínima, primando, por el contrario la mayor o menor exposición a otros agentes de degradación. (García, et al. 2009) 


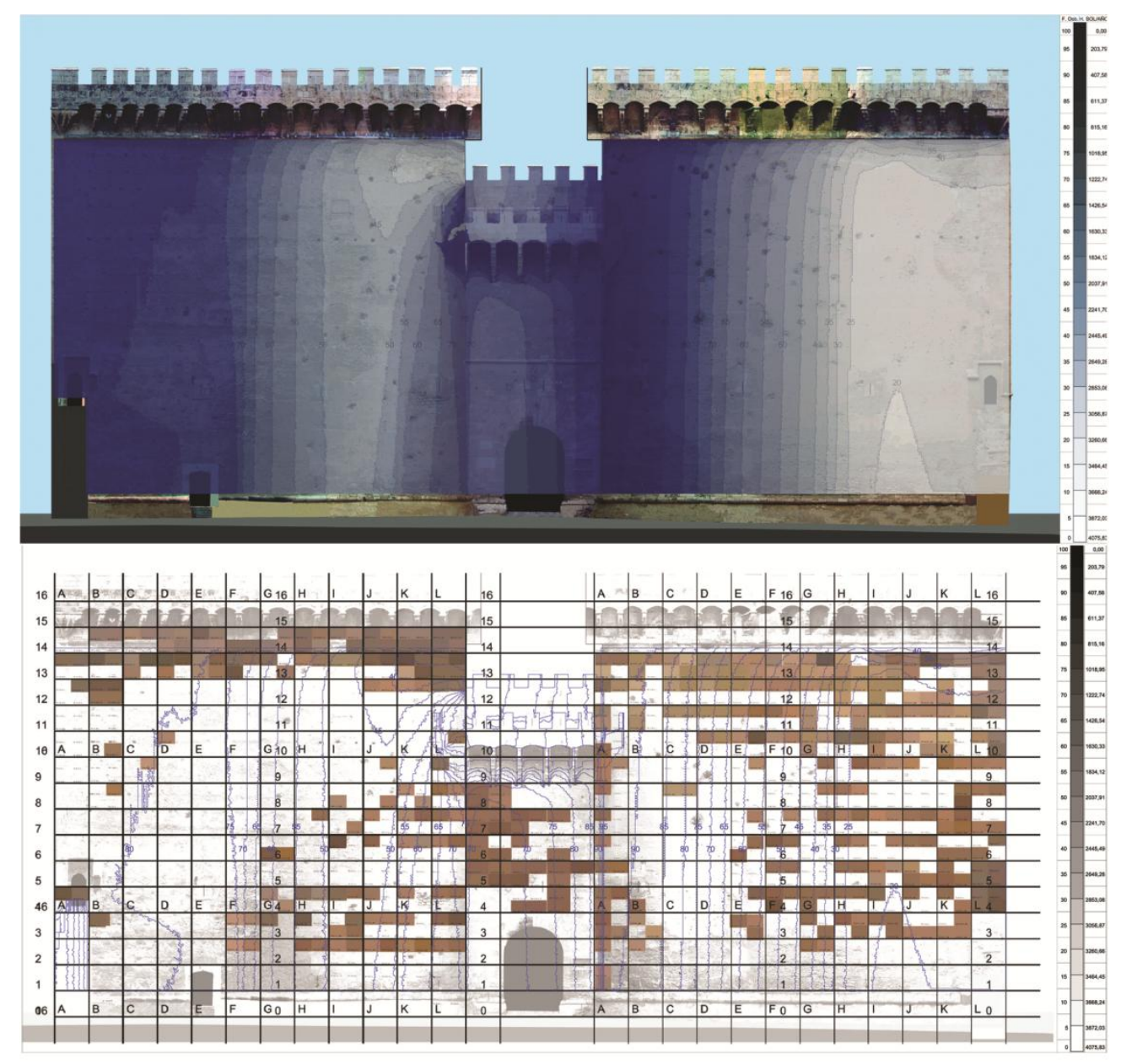

\section{Referencias}

Fairchild, M.D. (2005). "Color and Image Appearance Models". Color Appearance Models. p. 340, John Wiley and Sons.

García, A. Llopis, J. Masiá, J.V., Torres, A., Villaplana, R. (1995). El color del centro histórico: arquitectura histórica y color en el barrio del Carmen de Valencia. Ajuntament de València, Valencia.

García, A. Llopis, J., Torres, A., Villaplana, R. El color en el barrio de Velluters. (2000), Ajuntament de València, Valencia.

García, A., Llopis, J., Torres, A., Villaplana, R., Serra, J. (2009). "Colour as a structural variable of historical urban form". Color Research \& Application. Volume 34, Issue 3, pp. 253-265. Wiley InterScience. Hoboken (NY- United States)

Higón, J.L., Ferrer, J.J. (2003). Contribuciones al estudio del asoleo geométrico: procedimientos para el cálculo del factor de obstrucción solar: aplicaciones. Tesis doctoral, inédita, Universidad Politécnica de Valencia, Valencia.

Llopis, J., Torres, A., Serra, J., García. (2015). "The preservation of the chromatic image of historical cities as acultural value. The old city of Valencia (Spain)". Journal of Cultural Heritage. Paris. 
Martínez, M.J. (1990). Carta del restauro. Colegio de Arquitectos, Málaga.

Icomos. (1997) "Il restauro in italia e la carta di Venezia: atti del convegno". Edizioni Scientifiche Italiane, Napoli-Ravello.

Piles Selma V. (2007) Estudio de los morteros de los revestimientos continuos de las arquitecturas del centro histórico de valencia: preparación de morteros de restauración mixtos cal-puzolana. Tesis doctoral, inédita, Universidad Politécnica de Valencia, p. 221 Valencia.

Torres, A., Serra, J., Llopis, J., Higón, J. L., García, A., \& Sáiz, B. (2012). Análisis del color y el soleamiento en las Torres de Quart de Valencia (España). Informes de la Construcción, 64 (527), pp. 261-274.

Zaragoza A., Gómez-Ferrer, M. (2007). Pere Compte, Arquitecto. p. 428, Consorcio de Museos de la Comunitat Valenciana; Valencia. 